operators (or $n \times n$ matrices, by approximation), $\|p(U)\| \leqq 1$, so that $\|p(A)\| \leqq 1$ for all $A$ in $B$. By continuity, this holds for any function $p$ continuous in the closed unit disc, holomorphic in its interior, and satisfying (2). This was first proved by von Neumann [3] in 1951 (see also [2]).

\title{
REFERENCES
}

1. S. Bochner, Group invariance of Cauchy's formula in several variables, Ann. of Math. vol. 45 (1944) pp. 686-707.

2. E. Heinz, Ein v. Neumannscher Satz über beschränkte Operatoren im Hilbertschen Raum, Nachr. Akad. Wiss. Göttingen. Math.-Phys. Kl. IIa. (1952) pp. 5-6.

3. J. von Neumann, Eine Spektraltheorie für allgemeine Operatoren eines unitären Raumes, Math. Nachr. vol. 4 (1951) pp. 258-281.

Princeton University

\section{A PROOF OF LIOUVILLE'S THEOREM}

\section{EDWARD NELSON}

Consider a bounded harmonic function on Euclidean space. Since it is harmonic, its value at any point is its average over any sphere, and hence over any ball, with the point as center. Given two points, choose two balls with the given points as centers and of equal radius. If the radius is large enough, the two balls will coincide except for an arbitrarily small proportion of their volume. Since the function is bounded, the averages of it over the two balls are arbitrarily close, and so the function assumes the same value at any two points. Thus a bounded harmonic function on Euclidean space is a constant.

\section{Princeton University}

Received by the editors June 26, 1961. 